\title{
KNOWING THE NATURAL COURSE OF BIOMARKERS IN AD: LONGITUDINAL MRI, CSF AND PET DATA
}

\author{
W.M. VAN DER FLIER ${ }^{1}$, P. SCHELTENS
}

1. Associate professor, Alzheimer Center/ Dpt Neurology, VU University Medical Center, email: wm.vdflier@ vumc.nl, www.alzheimercentrum.nl

\section{Introduction}

In general, biomarkers can be used for two purposes: (i) to aid in the diagnosis of $\mathrm{AD}$, and (ii) to monitor disease progression. Clinical trials are in great need of biomarkers that reflect disease modification effects, to be used as end points. To date, we have a number of biomarkers in the field of MRI, CSF and PET that serve well as diagnostic marker. However, it is questionable if incorporating these biomarkers without second thought into clinical trials is useful. It is important to note, that biomarkers that have great value for diagnosis, may be of no use when it comes to monitoring disease progression. To monitor progression, we may have to look for a set of biomarkers that serves specifically this purpose. Furthermore, before such a marker can be usefully applied in clinical trials to demonstrate how a given intervention modifies the disease, it is of the utmost importance to have knowledge of naturalistic disease progression.

By definition, AD is a progressive disease, leading to increasing cognitive impairment, eventually resulting in loss of independence, institutionalization and death. The speed at which disease progression occurs however, varies considerably from patient to patient. Some patients show fast progression, whereas others remain relatively stable for many years. Studies on (determinants of) the natural course of the disease may prove to be very important.

1) Knowing what to expect from biomarkers in the natural course of the disease is a necessary prerequisite to start planning studies using these biomarkers. (i) knowledge of the natural course is necessary to be able to recognize a deviation from the natural course, (ii) knowledge on variability in the natural course is necessary to calculate sample sizes required to demonstrate a disease modification effect.

2) Knowing the determinants of speed of disease progression may have important implications for the design of trials; (i) selection of patient groups knowing to progress at a rapid rate enables demonstration of disease modifying effects over a short period, (ii) inclusion of patients that are expected to have a comparable natural disease course results in more homogeneous groups and consequently, larger power, and (iii) if rate of disease progression reflects subtypes of the disease, these may require different treatment approaches.

In this paper, we will describe data from studies investigating the natural course of MRI, CSF and PET biomarkers.

\section{Structural MRI}

Atrophy, especially of the medial temporal lobe is the radiological hallmark of $\mathrm{AD}$. Atrophy starts in the medial temporal lobe, gradually spreading to the rest of the cortex, eventually resulting in global atrophy. Medial temporal lobe atrophy is generally regarded as a sensitive marker for early diagnosis (1). For the purpose of diagnosis, global atrophy is a less established marker, although differences between AD and controls have been shown repeatedly $(2,3)$. For monitoring disease progression, the opposite may be true, as it seems that rate of globale atrophy may provide a more sensitive measure than rate of medial temporal atrophy.

To investigate the clinical use of hippocampal and whole brain atrophy rate, we included 65 patients with AD, 45 patients with MCI, and 37 controls (including patients with subjective complaints) (4, 5). Two MR scans were acquired, with an average interval of almost 2 years. Baseline volume and atrophy rates of both hippocampus and whole brain were measured. Hippocampal baseline volume and rate discriminated both MCI (hippocampal rate: $-3.8 \pm 1.2 \%$ ) and AD patients (rate: $-4.0 \pm 1.2 \%$ ) from controls (rate: $-2.2 \pm 1.4 \%, \mathrm{p}<0.05$ ), but did not discriminate $\mathrm{AD}$ from MCI. For whole brain, we found that baseline volume was lower in AD than in controls, but MCI could not be distinghuished from either group. Whole brain atrophy rate was the only measure that discriminated AD patients from MCI, as rates were higher in $\mathrm{AD}($ mean $\pm \mathrm{SD}$ $1.9 \pm 0.9 \% / y)$ than MCI $(-1.2 \pm 0.9 \% / y ; p<0.05)$, who in turn had higher whole-brain atrophy rates than controls $(-0.6 \pm 0.6 \% / y$; $\mathrm{p}<0.05)$. These results confirm that medial temporal lobe atrophy is the marker of choice regarding early diagnosis, but at the same time they suggest that medial temporal lobe may have reached a bottom level quite early in the disease process, rendering it less suitable as marker of progression once the stage of AD has been reached. By contrast, whole brain volume is less suitable as a marker of early diagnosis, but has the capacity to reflect disease progression throughout the disease. This was further illustrated by the fact that whole brain atrophy rate correlated with progressing disease severity as measured by change in mini-mental state examination (MMSE) $(r=0.48$, $\mathrm{p}<0.001)$.

We then investigated within the group of AD patients, which baseline clinical and MR measures influenced whole brain atrophy rates, to identify determinants of speed of disease progression (6). AD patients show an average whole brain 
volume loss of $2 \% / \mathrm{yr}$, but there is large variability, with patients losing as little as $1 \%$ per year, and much as $4 \%$ per year. We found that younger age ( $(\mathrm{SE})=0.03(0.01) ; \mathrm{p}=0.04)$, absence of APOE $4(\quad(\mathrm{SE})=0.61(0.28) ; \mathrm{p}=0.03)$, and a low baseline MMSE $(\quad(\mathrm{SE})=0.11(0.03) ; \mathrm{p}<0.001)$ were associated with a higher whole-brain atrophy rate. Furthermore, a relatively spared hippocampus predicted faster decline for patients with a smaller baseline brain volume, and with a lower MMSE. These results suggest that it is possible to characterise a subgroup of $\mathrm{AD}$ patients, that are at risk of faster loss of brain volume. Patients with more generalized, rather than focal hippocampal atrophy, who often have an onset before the age of 65 , and are APOE 4 negative, seem to be at risk of faster whole-brain atrophy rates than the more commonly seen $\mathrm{AD}$ patients, who are older, APOE 4 positive and have pronounced hippocampal atrophy.

\section{CSF}

In CSF, decreased levels of amyloid beta 1-42 (A 42) and increased levels of tau and phosphorylated tau are thought to reflect the presence of AD pathology (7). These CSF biomarkers have been shown to be of diagnostic use, as they differentiate patients with $\mathrm{AD}$ from control subjects with reasonable accuracy (8). Moreover, these changes can be detected in patients with mild cognitive impairment (MCI) who will progress to $\operatorname{AD}(9,10)$. As with the abovementioned MRI markers, it is not a given fact that biomarkers that have good value as diagnostic markers, are equally usable to monitor progression.

To investigate the use of CSF biomarkers as markers of progression, we performed repeated lumbar puncture in 105 patients (50 AD, $38 \mathrm{MCI}, 17$ subjective complaints), with an average interval of almost two years. CSF levels of A 42, tau and ptau-181 were measured with a sandwich ELISA (Innogenetics). We started by assessing the variability of measuring change over time $(11,12)$. Baseline samples were assayed twice: once shortly after the first spinal tap and once, in a separately stored aliquot, concomitant with the follow-up sample. Variances of coefficients of variation were calculated. We found that variance of the baseline and follow up CSFsample pairs run on separate assays was considerably larger than for pairs run on the same assay. Variance of repeated assessment of the same baseline samples in different assays tended to be larger than combined assessment of baseline and follow-up sample in one assay. We concluded that in case of repeated spinal taps, determination of CSF biomarker levels should be performed in the same assay. Although we found considerable variability, others have reported a marked stability over time of these biomarkers in AD patients (13).

We than evaluated the natural course of CSF biomarkers in patients with $\mathrm{AD}$, subjective complaints and mild cognitive impairment (MCI) (14). CSF A 1-42 and tau, but not ptau-181 levels increased over time in this memory clinic patient cohort with comparable change in all diagnostic groups. The crosssectional difference between diagnostic groups, however, exceeded by far the longitudinal changes within individuals, suggesting that these biomarkers are unsuitable as markers of disease progression. Moreover, we showed that change in CSF biomarker levels was not related to either change in MMSE or atrophy rate (15). Contrasting with these findings, a recent study showed change over time in CSF levels of ptau-181, but not A 1-42 and tau in a group of progressive MCI patients (16).

Like the MRI markers, it is questionable if the CSF biomarkers that have clearly been proven useful for the diagnostic work-up of $\mathrm{AD}$, are the markers of choice for monitoring disease progression. When we cannot yet track the naturalistic disease course using CSF biomarkers, it seems unfeasible to demonstrate a modified disease course. In theory, it is still possible that the existing biomarkers would reflect a large reduction in Alzheimer pathology burden, but even then, it is important to keep in mind that the measurement errors in the existing biomarkers are large. Possibly, alternative biomarkers, such as isoprostanes, are more suitable to measure disease progression.

\section{PET}

Literature on amyloid imaging using PET is quickly accumulating. It seems that [11C]PIB accurately reflects amyloid deposition, and that PIB discriminates diagnostic groups well $(17,18)$. MCI patients seem to be categorized in PIB positive and PIB negative patients, related to subsequent progression to AD (19). Studies reporting on change in PIB binding over time are scarce. The few available studies seem to suggest that PIB imaging reflects an on/off phenomenon, and hardly show change over time, in line with findings in CSF A 42 , and with post mortem studies that do not show a relation between plaque load and disease severity. FDDNP is an alternative tracer that claims to visualize amyloid load (20). Although the diagnostic value of FDDNP is less established, it seems that it has at least some value, measuring related but different aspects of the disease $(21,22)$. The discriminatory value of FDDNP is clearly less than that of PIB, but it is possible that FDDNP has more potential as a marker of disease progression (20).

\section{Conclusion}

To sum up, as a biomarker of naturalistic disease progression, whole brain atrophy rate seems to be the marker of choice at this moment. It is a robust measure, that discriminates between groups, and is related to clinical progression. Hippocampal atrophy performs better as a diagnostic marker, and possibly as a marker of progression in the earliest disease stages, i.e. MCI. The three most widely known CSF biomarkers of $\mathrm{AD}$ do not seem useful as markers to monitor disease 


\section{JNHA: CLINICAL TRIALS AND AGING}

progression with the current methods. Finally, data on longitudinal amyloid imaging using PET are still awaited. PIB is expected to be of little or no use to monitor disease progression. It is still possible that FDDNP, although less useful in the diagnosis of AD may prove of value to track disease progression. paper.

Financial disclosure: None of the authors had any financial interest or support for this

\section{References}

1. Scheltens P, Fox N, Barkhof F, De Carli C. Structural magnetic resonance imaging in the practical assessment of dementia: beyond exclusion. Lancet Neurol 2002; 1(1):13-21.

2. Wolf H, Grunwald M, Kruggel F, Riedel-Heller SG, Angerhofer S, Hojjatoleslami A et al. Hippocampal volume discriminates between normal cognition; questionable and mild dementia in the elderly. Neurobiol Aging 2001; 22(2):177-186.

3. Petersen RC, Jack CRJ, Xu YC, Waring SC, O'Brien PC, Smith GE et al. Memory and MRI-based hippocampal volumes in aging and AD. Neurology 2000; 54:581587.

4. Sluimer JD, van der Flier WM, Karas GB, Fox NC, Scheltens P, Barkhof F et al. Whole-brain atrophy rate and cognitive decline: longitudinal MR study of memory clinic patients. Radiology 2008; 248(2):590-598.

5. Henneman W.J., Sluimer JD, Barnes J, van der Flier WM, Sluimer IC, Fox N et al. Hippocampal atrophy rates in Alzheimer's disease: added value over whole brain volume measures. Neurology. 2009;(In Press)

6. Sluimer JD, Vrenken H, Blankenstein MA, Fox NC, Scheltens P, Barkhof F et al. Whole-brain atrophy rate in Alzheimer disease: identifying fast progressors. Neurology 2008; 70(19 Pt 2):1836-1841.

7. Blennow K, Hampel H. - CSF markers for incipient Alzheimer's disease. - Lancet Neurol 2003 Oct;2(10):605-13(- 1474-4422 (Print))

8. Wiltfang J, Lewczuk P, Riederer P, Grunblatt E, Hock C, Scheltens P et al. Consensus paper of the WFSBP Task Force on Biological Markers of Dementia: the role of CSF and blood analysis in the early and differential diagnosis of dementia. World J Biol Psychiatry 2005;6(2):69-84(- 1562-2975 (Print)).

9. Bouwman FH, Schoonenboom SNM, van Der Flier WM, Van Elk EJ, Kok A, Barkhof $\mathrm{F}$ et al. CSF biomarkers and medial temporal lobe atrophy predict dementia in mild cognitive impairment. Neurobiol Aging 2007; 28(7):1070-1074.

10. Hansson O, Zetterberg H, Buchhave P, Londos E, Blennow K, Minthon L. Association between CSF biomarkers and incipient Alzheimer's disease in patients with mild cognitive impairment: a follow-up study. - Lancet Neurol 2006 Mar;5(3):228-34(- 1474-4422 (Print)).

11. Bouwman FH, van der Flier WM, Schoonenboom NS, Van Elk EJ, Kok A, Scheltens $P$ et al. Usefulness of Longitudinal Measurements of Beta-Amyloid1-42 in Cerebrospinal Fluid of Patients with Various Cognitive and Neurologic Disorders. Clin Chem 2006; 52(8):1604-1606.

12. Verwey NA, Bouwman FH, van der Flier WM, Veerhuis R, Scheltens P, Blankenstein MA. Variability in longitudinal cerebrospinal fluid tau and phosphorylated tau measurements. Clin Chem Lab Med 2008; 46(9):1300-1304.

13. Blennow K, Zetterberg H, Minthon L, Lannfelt L, Strid S, Annas P et al. Longitudinal stability of CSF biomarkers in Alzheimer's disease. Neurosci Lett 2007; 419(1):18-22.

14. Bouwman FH, van der Flier WM, Schoonenboom NS, Van Elk EJ, Kok A, Rijmen F et al. Longitudinal changes of CSF biomarkers in memory clinic patients. Neurology 2007; 69(10): 1006-1011

15. Sluimer JD, Bouwman FH, Vrenken H, Blankenstein MA, Barkhof F, van der Flier $\mathrm{WM}$ et al. Whole-brain atrophy rate and CSF biomarker levels in $\mathrm{MCI}$ and $\mathrm{AD}$ : A longitudinal study. Neurobiol Aging 2008.

16. Andersson C, Blennow K, Almkvist O, Andreasen N, Engfeldt P, Johansson SE et al. Increasing CSF phospho-tau levels during cognitive decline and progression to dementia. Neurobiol Aging 2008; 29(10):1466-1473.

17. Klunk WE, Engler H, Nordberg A, Wang Y, Blomqvist G, Holt DP et al. Imaging brain amyloid in Alzheimer's disease with Pittsburgh Compound-B. Ann Neurol 2004; 55(3):306-319.

18. Edison P, Archer HA, Hinz R, Hammers A, Pavese N, Tai YF et al. Amyloid, hypometabolism, and cognition in Alzheimer disease: an [11C]PIB and [18F]FDG PET study. Neurology 2007; 68(7):501-508.

19. Jack CR, Jr., Lowe VJ, Senjem ML, Weigand SD, Kemp BJ, Shiung MM et al. 11C $\mathrm{PiB}$ and structural MRI provide complementary information in imaging of Alzheimer's disease and amnestic mild cognitive impairment. Brain 2008; 131(Pt 3):665-680.

20. Small GW, Kepe V, Ercoli LM, Siddarth P, Bookheimer SY, Miller KJ et al. PET of brain amyloid and tau in mild cognitive impairment. N Engl J Med 2006; 355(25):2652-2663.

21. Shin J, Lee SY, Kim SH, Kim YB, Cho SJ. Multitracer PET imaging of amyloid plaques and neurofibrillary tangles in Alzheimer's disease. Neuroimage 2008; 43(2):236-244.

22. Tolboom N, Yaqub M, van der Flier WM, Boellaard R, Luurtsema G, Windhorst A et al. Detection of Alzheimer pathology in vivo using both [11C]PIB and [18F]FDDNP positron emission tomography. J Nucl Med. 2009;(In Press) 